\title{
A Centennial of Protons
}

In the modern and exciting world of particle physics, in which scientist talk of Higgs bosons and supersymmetry, it would be natural for someone to dismiss the common proton as a particle too pedestrian to be interesting. Yet in the centennial year of the announcement of its discovery, studies of the humble nucleus of the hydrogen atom continue to teach us fascinating lessons about the subatomic world.

As recently as 2018 , scientists found themselves unable to definitively determine as simple a parameter as the radius of the proton. And uncertainties in the detailed internal structure of the proton continue to be the dominant limitation of precision measurements conducted at such particle accelerators as the Large Hadron Collider. Indeed, the final story of the proton has yet to be told.

\section{History}

As familiar as the proton is, it's valuable to remember that it wasn't all that long ago that even its existence wasn't known to science [1,2]. Ernest Rutherford is most famously known for his experiments shooting alpha particles at a thin gold film, which resulted in the then-surprising observation that some of the alpha particles ricocheted backward, "as if a 15-inch shell had bounced off a sheet of tissue paper." J.J. Thomson, Rutherford's thesis advisor and discoverer of the electron, had proposed what is called the Plum Pudding model of the atom, in which tiny and negatively charged electrons were embedded in some sort of positively charged goo.

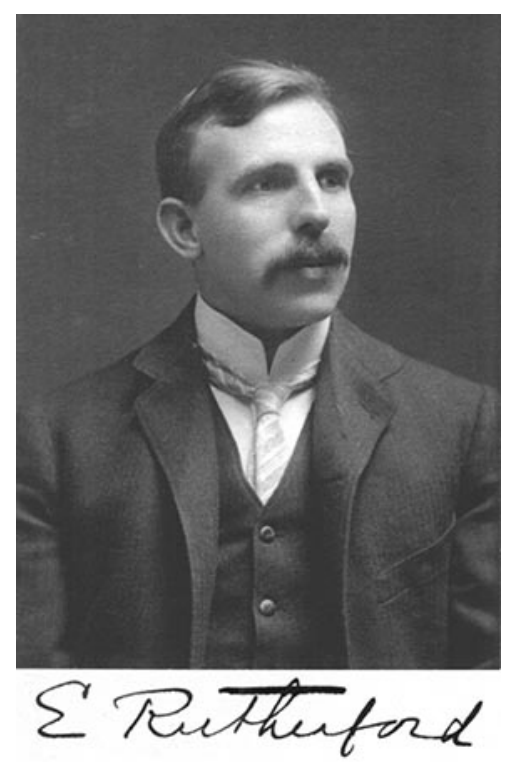

Figure 1: New Zealand physicist Ernest Rutherford was a key contributor to our understanding of the structure of both atoms and the atomic nucleus.

However, Rutherford's experiment proved Thomson's model was incorrect and, after a year or so thinking about the implications of his experiment, Rutherford realized that 
atoms consisted of a small and dense positively charged core, surrounded by a diffuse cloud of electrons.

But the nature of the nucleus of the atom was not immediately apparent. In fact, there was a school of thought that treated atomic nuclei as objects that were not able to be split into smaller units.

It was in 1913 that Rutherford directed his assistant Ernest Marsden to "play marbles" with alpha particles and light nuclei, especially hydrogen nuclei. From simple classical calculations (of a one-dimensional collision with the proton initially at rest and by using a statistical treatment of a charged particle slowing in matter from repeated collisions with atomic electrons), one can determine that in a head-on collision between an alpha particle and a hydrogen nucleus (called at the time "H" particles, but what we now call the proton), the nucleus should recoil with a speed 1.6 times that of the alpha particle and penetrate material to a depth four times deeper than the initial alpha [3]. Marsden did indeed see $\mathrm{H}$ particles with the appropriate range.

However, Marsden also saw $\mathrm{H}$ particles when alpha particles were passed through air. Where those particles came from was not known, whether it was the air itself, water vapor, or some contaminant.

World War I intervened, and Rutherford turned his attention to submarine detection and it wasn't until 1917 that he returned to experiments involving alpha particles. He continued to shoot alpha particles at a variety of materials, including hydrogen, hydrogen-rich solids, carbon dioxide, and nitrogen. He found that in alpha/nitrogen collisions that he saw a lot of $\mathrm{H}$ particle emission. He deduced that what was happening was, that in the collision, $\mathrm{H}$ particles were being knocked off the nitrogen nucleus. From that insight, it was a short intellectual step to propose that atomic nuclei were made of an assemblage of hydrogen nuclei. And, in 1919, he announced his conclusions to the world. It was in 1920 that Rutherford coined the term "proton."

Rutherford also hypothesized that there existed in the nucleus of atoms another, electrically-neutral, particle, with a mass similar to the proton. He suggested that James Chadwick, a student of his, investigate this hypothesis and Chadwick discovered the neutron about a decade later. The triumvirate of advisor, researcher, and student (Thomson, Rutherford, and Chadwick) had unraveled the structure of the atom.

\section{Properties}

In short order, the properties [4] of the proton were determined. It has an electrical charge of $1.602176634 \times 10^{-19}$ coulombs, equal in magnitude, but opposite in sign to the electron. Precisely why these two subatomic particles have exactly the same magnitude is still unknown. The proton has a mass of $1.007276466879 \pm$ $0.000000000091 \mathrm{amu}$, or $938.2720813 \pm 0.0000058 \mathrm{MeV} / \mathrm{c}^{2}$. Its radius, as defined by the distribution of electrical charge, is about $0.85 \mathrm{fm}$, although two measurements, using different techniques and both quoting very precise uncertainties, are in disagreement. 
More will be said of that below.

The lifetime of the proton, including all decay modes, is $>2.1 \times 10^{29}$ years, with an estimated lifetime of the theoretically-attractive decay mode of $\mathrm{p}^{+} \rightarrow \mathrm{e}^{+} \pi^{\circ}$ of $>8.2 \times 10^{33}$ years. (The lower limit on the proton's lifetime reflects limited experimental sensitivity to all possible decay modes, while the much higher limit on the single decay chain reflects increased instrumental sensitivity to that particular decay mode.) The proton has a spin of $\hbar / 2$, where $\hbar$ is the reduced Planck's constant. The proton has a magnetic moment of $2.79284734462 \pm 0.00000000082$ Bohr magnetons and an electric dipole moment of $<$ $0.021 \times 10^{-23} \mathrm{e} \mathrm{cm}$, which means that it is incredibly spherical.

With such precise measurements of the properties of this well-known particle, it would seem that the proton would hold few mysteries. However, the proton is far more complex than the simplified version that plays a role an atomic and nuclear physics.

\section{Proton Structure: Quarks}

The 1950s were an exciting time for particle physics. By converting energy into mass, researchers used particle accelerators to create subatomic particles that were not typically found in nature. These particles had a diverse set of properties, but a subset called baryons were qualitatively similar to the familiar proton and neutron (e.g. similar in mass and experienced the strong nuclear force). In addition, there were the lighter mesons, which were superficially similar to the proton and neutron, but with a different subatomic spin. Mesons are all bosons, with integer spin in units of $\hbar$, while the baryons were fermions, with half integer spin. For over a decade, researchers grappled with the patterns of charges, masses, lifetimes, and other properties until 1964 when Murray Gell-Mann and George Zweig independently realized $[1,5]$ that the patterns could be explained if protons contained smaller particles within them. Gell-Mann called them "quarks," while Zweig used the term "aces." Gell-Mann's choice has been adopted by the scientific community. 


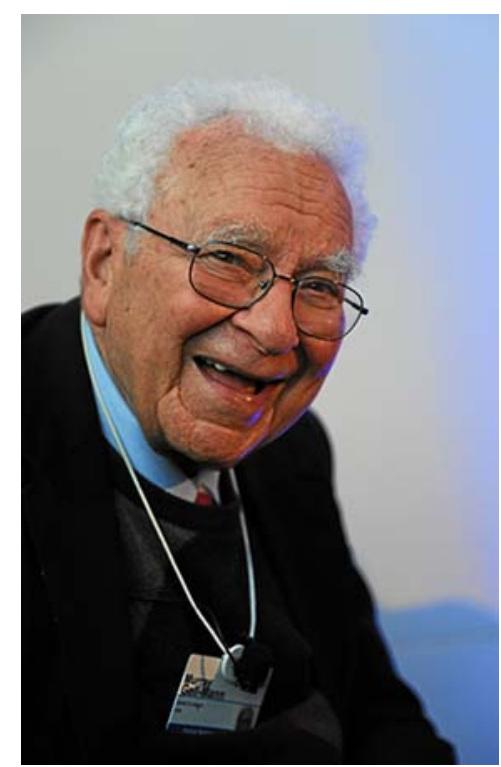

Figure 2: American physicist Murray Gell-Mann proposed the quark model in 1964.

In Gell-Mann's initial paper, three distinct varieties of quarks were proposed, with the names up, down, and strange. The names have historical significance, with up and down connected to nuclear isospin [6], and strange having to do with a conserved quantity observed in the production of certain baryons [1]. Since the model was initially proposed, three additional quarks have been discovered, called charm, bottom, and top.

The up, charm, and top quarks have an electrical charge of $+2 / 3$ that of a proton, while the down, strange, and bottom quarks have a charge of $-1 / 3$ that of a proton. All quarks are fermions with spin of $\pm 1 / 2$ in units of $\hbar$.

Baryons contain three quarks, with the proton containing two up quarks and one down quark. Neutrons contain one up quark and two down quarks. In order for the fermion quarks to create a fermion proton with a spin of $1 / 2$, the spin of the quarks must be oriented with two parallel and one antiparallel.

Baryons contain any combination of quarks and, at the time the model was proposed, the configuration containing three strange quarks had not been observed. However, very shortly after the quark model was proposed, the $\Omega^{-}$baryon was discovered [7]. This particle contained three strange quarks and thus the quark model was validated.

The $\Omega^{-}$baryon posed a problem for physicists. It has a spin of $3 / 2$ [8], which means that it has three strange quarks, all with spin of $1 / 2$. Quantum mechanics forbids identical fermions to exist in the same quantum state [9], so this particle runs afoul of very basic physical principles.

This problem was resolved in 1964 by Oscar Greenberg, although his treatment differed from a modern methodology and Greenberg has mentioned Han and Nambu as an improved approach [10]. These researchers proposed a new quantum number called 
"color" as a way to distinguish between the three quarks. Each quark has a unique color (red, green, blue), while the proton as a whole has none (e.g. white). The term color has nothing to do with color as the word is generally understood, but it mirrors the property of red, blue and green light to appear white when mixed.

\section{Proton Structure: Partons}

While the quark model made some predictions, many physicists, including Gell-Mann, thought quarks as representing an organizing mathematical structure and were not actual particles. That view started to change in 1968 when data recorded using the SLAC accelerator began to reveal that protons were definitely composite particles [11].

These experiments shot high energy electrons at a stationary proton target. In principle, the kinematics of the collisions between an electron and point-like proton is simple, twobody, elastic scattering, governed by the same mathematics as taught in any introductory physics course. Further, by simply measuring the energy of the incoming and outgoing electron and assuming the proton was at rest, the kinematics of the collision are fully determined.

When researchers performed these experiments (and eventually with follow-on experiments using both muons and neutrinos as beam particles), they found that the simplest prediction did not work. While collisions at lower energy followed the predictions of electron-proton elastic collision theory, when the collisions became more violent, the collisions became increasingly inelastic. The data very definitely suggested that protons contained constituent particles that carried a fraction of the energy and momentum of the proton.

In 1969, Richard Feynman coined the term "partons" to describe these quark constituents [12] and his approach was followed by James Bjorken and Emmanuel Paschos in interactions between electrons and protons.

The SLAC experiment revealed that protons contained many particles that interacted with one another. Further, they demonstrated that proton contained more than the three quarks postulated by Gell-Mann. The measurement determined the proton consisted of many partons, each carrying a fraction $x$ of the momentum of the proton, e.g. $x=$ $p_{\text {parton }} / p_{\text {proton. }}$

In each collision, an electron passed near the proton and emitted a photon that probed deeply inside the proton and interacted with one of the electrically charged partons. Each collision interacted with a single parton and, event after event, researchers were able to reconstruct the distribution of the fraction of the momentum carried by electrically charged partons. Further, this measurement reveals a great deal about the distribution of momentum within protons.

Figure 3 illustrates this point. If the photon emitted by the electron interacted with a solid and structureless particle, the momentum fraction would always be simply 1 . In 
the Gell-Mann non-interaction quark model, each of the three quarks would carry precisely $1 / 3$ of the proton's momentum. If the proton's constituent quarks were able interact with one another, we would expect a momentum fraction distribution peaked near $1 / 3$, but with some variation. And if the quarks not only exchanged momentum, but also emitted force-carrying particles that could then subsequently convert into quark matter/antimatter pairs, the distribution would be further modified to have more of the proton's momentum concentrated at very low momentum fraction. It is this fourth possibility that is what is observed.

These observations led to the development of the theory of quantum chromodynamics, which is the model of strong nuclear force interactions $[1,15]$. Briefly, in this model, a proton consists of three "valence" (e.g. persistent) quarks as predicted by Gell-Mann, but the force between the quarks is mediated by the exchange of force carrying particles called gluons. These gluons can briefly turn into quark matter/antimatter pairs (called "sea" quarks), before they annihilate and become a gluon which is then absorbed by other quarks. The structure of a proton is extremely complicated.

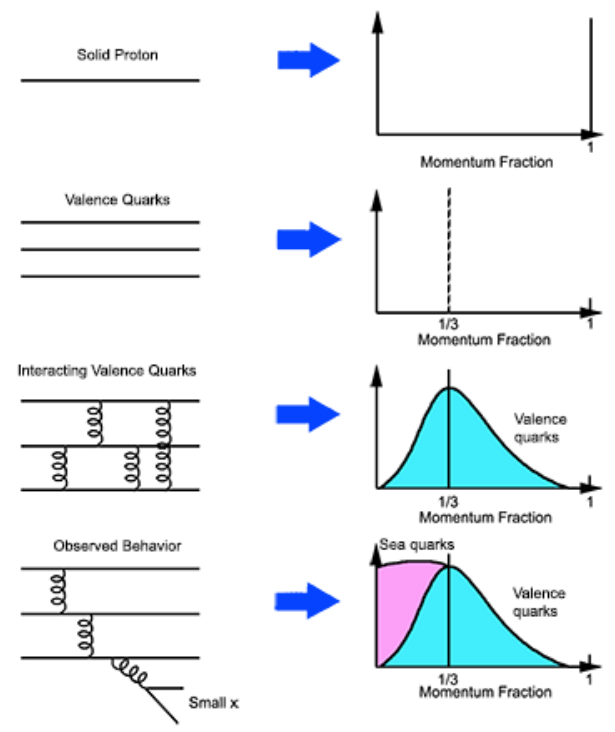

Figure 3: The distribution of fractional momentum within the proton depends on the proton's internal structure. The different scenarios are described in the text. Figure inspired by [14].

The structure of the proton is further complicated by the fact that there is a large range of the physical size of those structures. Because the wavelength of the probe photon is inversely proportional to its momentum, higher momenta photons can resolve smaller structures, as seen in figure 4. Accordingly, the structure of the proton becomes more complex and a larger fraction of the proton's momentum can be seen to be stored in low-momentum, small-size structures. Physicists must thus not only study the structure of the photon as a function of the momentum fraction they are investigating, but also as a function of the scale of the wavelength of the probing photon. Further, it is possible to make a measurement at one momentum fraction and photon wavelength scale and extrapolate to other scales. 
An accurate knowledge of the distribution of momentum among partons is crucial for accurate predictions of high energy experiments. Of special concern is the distribution of momentum carried by gluons at high-x. Because gluons (being neutral and not subject to either the electromagnetic or weak forces) cannot be directly probed by electron, muon, or neutrino beams, this distribution remains relatively poorly measured. It can only be investigated in collisions involving pairs of hadrons. These measurements have constrained this distribution, but further work is needed.
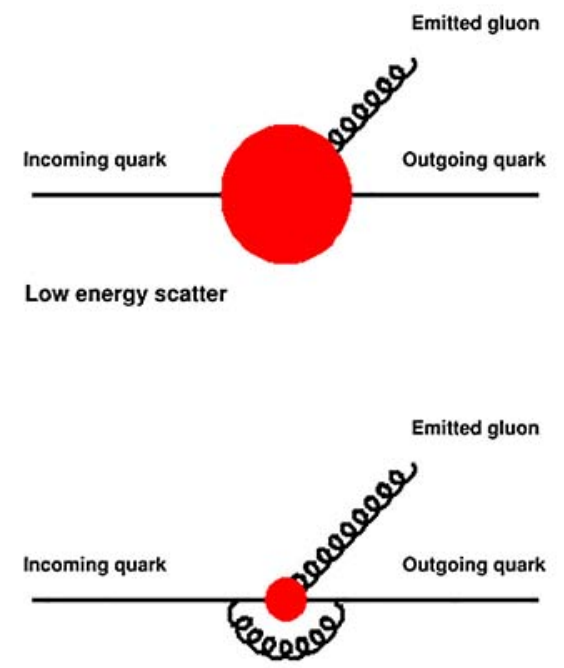

High energy scatter

Figure 4: When the structure of protons is studied in high energy collisions, more detailed structures can be resolved.

\section{Proton Spin}

A proton is a fermion with spin $1 / 2$. In the simplest quark model, it contains three fermion quarks of spin $1 / 2$. Accordingly, in the quark model, the spin of the proton resides in the alignment of its constituent valence quarks, with two quarks having parallel spin and one antiparallel. However, in 1988, the European Muon Collaboration (EMC) fired a beam of muons with known spin polarization at a target of polarized protons (e.g. ones where the spin of the protons are aligned) and measured the spin of the proton carried exclusively by the intrinsic spin of the quarks and antiquarks, and found that they amounted to only a fraction of the spin of the proton. Essentially, they found that the spin of the quarks and antiquarks were (on average) equally parallel and antiparallel to the proton [16].

The EMC experiment used a muon beam, which emitted a photon, which then interacted exclusively with the charged partons (e.g. quarks and antimatter quarks) in the proton. EMC was not able to study the contribution to the spin of the proton from gluons. Gluons are spin 1 bosons.

It took two decades before spin information for gluons began to become available. These measurements arose from data recorded from collisions between two polarized beams of protons at the Relativistic Heavy lon Collider (RHIC) at Brookhaven National 
Laboratory. In 2008, the first studies revealed a gluonic contribution to the spin of the proton. However, there were large experimental uncertainties and it took yet another decade to get a better picture of what is going on. More recent measurements [17] show that the spin of a proton comes from a very complicated admixture of the intrinsic spin of the both the valence quarks and sea quark/antiquark pairs, as well as their orbital angular momentum. In addition, the gluons contribute spin from both their intrinsic and orbital motion. The specific fractions from each component continue to be studied at locations, which include the Thomas Jefferson National Accelerator Facility, in Newport News, VA.

Figure 5 illustrates the differences between our understanding of the structure of the proton in 1980 compared to it now. This figure highlights the color (e.g. strong force charge) of the proton, along with a simplified (but modern) depiction of how the proton spin is distributed among the valence quarks, the sea quarks and the motion of the partons, including the gluons. This figure should be contrasted to the depiction of the proton on the cover of this issue. In that artistic rendition, representation of the strong force charge and the spin is entirely missing. Instead, that image focuses on the flavor (e.g. quark type) of the partons. There, the blue spheres represent up quarks and the red ones denote down quarks. The smaller spheres represent quark/antiquark sea pairs, with the ones with a highlighted rim being the antiquarks. And in the cover image, the dashed lines give the smallest flavor of the cacophony of gluons that are constantly jumping throughout the proton. The contrast between the cover and figure 5 demonstrates the difficulty of illustrating the entire complexity of the partonic content of the proton, as it contains a variety of properties, including particle flavors, strong force charge (color), electrical charges, spins, motion, and both matter and antimatter components. The structure of the proton is exceedingly rich.

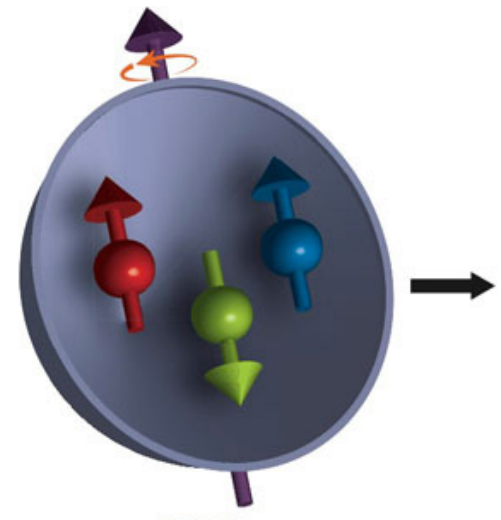

$1980 s$

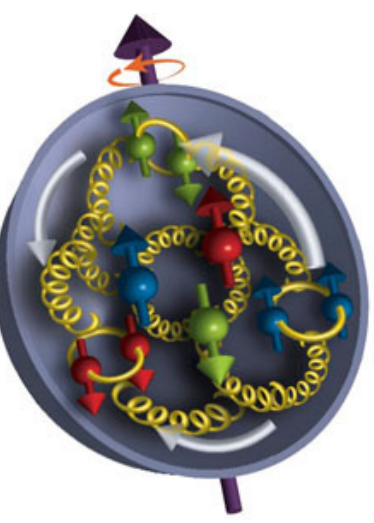

now

Figure 5: As late as the early 1980s, our understanding of the origin of spin in the proton rested in the spin of the valence quarks. However, we now realize that the spin rests in both the intrinsic spin and orbital motion of all of the proton's constituents. (Figure courtesy Brookhaven Lab.)

\section{Proton Radius}


To determine the size of a proton, one first needs to have an accurate mental image of the particle. Protons are not hard objects, like bowling balls. Instead, the surface of a proton is more analogous to the Earth's atmosphere, denser near the surface of the Earth and more rarified at larger distances. This distribution has been probed by scattering electrons off protons and a root mean square radius of the charge distribution of a proton has been determined to be about $0.88 \mathrm{fm}$. Since about the year 2,000, other studies have employed very precise measurements of spectral transitions in electrons surrounding the proton to extract a precise measurement of the proton's radius. Because some atomic orbitals extend to the center of the atom, these transitions are sensitive to the charge distribution of protons. When all experiments of these nature are combined, the RMS charge radius of the proton is $0.8751 \pm 0.0061 \mathrm{fm}[4]$.

Measurements of the spectral transitions in muonic hydrogen (e.g. atoms in which the electron is replaced by a muon) are also sensitive to the RMS charge radius of the proton. Further, because muonic hydrogen is $0.5 \%$ the size of regular hydrogen, these measurements are more sensitive to the charge distribution of the proton. These measurements result in a different number, specifically $0.84087 \pm 0.00039 \mathrm{fm}$ [4]. These numbers disagree in a statistically significant way and this is called the protonradius puzzle.

Initially, the solution to the puzzle was thought to arise from differences between electrons and muons and there was the exciting prospect that perhaps new physical phenomena might be the cause. However more recent measurements [18] of the size of proton using ordinary hydrogen (i.e. protons + electrons) have resulted in a lower estimate for the proton's radius. It is appearing that the "electron vs. muon" solution is not the answer.

The problem appears to arise between atomic and scattering measurements and it may well be that the disagreement is rooted in limitations in the scattering technique. In scattering experiments, the proton recoils against the probing particle and this must be taken into account. At the moment, experimental groups must make measurements as a function of the energy of the probe and extrapolate to zero scattering energy.

Recent measurements of the radius of the proton are now available at much lower collision energies, and they report a smaller proton radius of $0.810 \pm 0.082 \mathrm{fm}$ [19]. Other low energy measurements report a similarly-low measurement of the proton's radius [20]. Additional studies are currently under preparation or underway [21] at a variety of laboratories around the world and it would appear that a future world average estimate of the radius of the proton will be smaller than that reported in [4].

\section{Closing remarks}

The proton is one of the fundamental building blocks of atomic matter and we've known of its existence for a century. Yet the reality is that it remains an interesting particle, with many mysteries still to be resolved. It is fitting that, in its centennial year, we can wish that studies of the proton will continue for years to come. Happy Birthday proton! 


\section{References}

[1] D. Lincoln, Understanding the Universe: From Quarks to the Cosmos, World Scientific, 2014 (revised); R. Crease and C. Mann, The Second Creation: Makers of the Revolution in Twentieth-Century Physics, Rutgers University Press, 1996; L. Lederman, D. Teresi, God Particle: If the Universe is the Answer, What is the Question?, Mariner Books, (2006).

[2] J. Campbell, Rutherford, Transmutation and the Proton, CERN Courier, May/June 2019, p. 27 (https://cerncourier.com/rutherford-transmutation-and-the-proton/).

[3] M. Tanabashi et al. (Particle Data Group), Phys. Rev. D 98, 030001 (2018) (http://pdg.lbl.gov/2019/reviews/rpp2018-rev-passage-particles-matter.pdf). Note that the velocity of the outgoing proton is determined by assuming a onedimensional elastic scattering between an alpha particle $\left(m_{\alpha}=4 m_{p}\right)$, moving at velocity $\mathrm{v}$, hitting a stationary proton $\left(\mathrm{m}_{\mathrm{p}}\right)$. The depth of penetration is determined by using equation 33.5 in this reference.

[4] M. Tanabashi et al. (Particle Data Group), Phys. Rev. D 98, 030001 (2018) (http://pdg.lbl.gov/2018/listings/rpp2018-list-p.pdf).

[5] M. Gell-Mann, A Schematic Model of Baryons and Mesons, Phys. Lett. 8 (3) (1964) p. 214; G. Zweig, An SU(3) Model for Strong Interaction Symmetry and its Breaking, CERN Report No. 8182/TH.401, (1964).

[6] D. Griffiths, Introduction to Elementary Particles, Wiley-VCH (2 ${ }^{\text {nd }} \mathrm{Ed}$. ) 2008.

[7] V.E. Barnes, et al., Observation of a Hyperon with Strangeness Minus Three, Phys. Rev. Lett. 12, 204.

[8] B. Aubert et al. (BABAR Collaboration), Measurement of the Spin of the $\Omega^{-}$Hyperon, Phys. Rev. Lett. 97, 112001.

[9] K. Krane, Modern Physics, Wiley (3rd Ed.), (2012).

[10] O. Greenberg, Spin and Unitary Spin Independence in a Paraquark Model of Baryons and Mesons, Phys. Rev. Lett. 13, 598-602 (1964); M.Y. Han and Y. Nambu, Three-Triplet Model with Double SU(3) Symmetry, Phys. Rev. 139 (1965) B1006.

[11] E. D. Bloom, et al., High-Energy Inelastic e-p Scattering at $6^{\circ}$ and $10^{\circ}$, Phys.I Rev. Let. 23 (16): 930-934; M. Breidenbach, et al., Observed Behavior of Highly Inelastic Electron-Proton Scattering, Phys. Rev. Lett. 23 (16): 935-939.

[12] R. Feynman, The Behavior of Hadron Collisions at Extreme Energies, High Energy Collisions: Third International Conference at Stony Brook, N.Y., (1969) pp. 237-249.

[13] J. Bjorken and E.Paschos, Inelastic Electron-Proton and $\gamma$-Proton Scattering and the Structure of the Nucleon, Phys. Rev. 185 (5): 1975.

[14] F. Halzen and A. Martin, Quarks and Leptons: An Introductory Course in Modern Particle Physics, Wiley, (1984). (Figure 3 from page 201.)

[15] D.J. Gross and F. Wilczek, Ultraviolet behavior of non-abelian gauge theories, Phys. Rev. Let. 30 (26): 1343-1346; H.D. Politzer, Reliable perturbative results for strong interactions, Phys. Rev. Let. 30 (26): 1346-1349.

[16] R. Jaffe, Where does the proton really get its spin?, Physics Today. 48 (9): 24-30 (1995). 
[17] J. Adam, et al. (STAR Collaboration), Measurement of the longitudinal spin asymmetries for weak boson production in proton-proton collisions at $\sqrt{ } \mathrm{s}=510 \mathrm{GeV}$, Phys. Rev. D 99, 051102(R).

[18] A. Beyer, et al., The Rydberg constant and proton size from atomic hydrogen, Science, 35879.

[19] M. Mihovilovič, et al., First measurement of proton's charge form factor at very low $Q^{2}$ with initial state radiation, Phys. Lett. B771 (2017) 194.

[20] As of this writing, these measurements have not been published and exist only in unpublished conference talks.

[21] M. Aghasyan, et al. (COMPASS Collaboration), First Measurement of the Transverse-Spin-Dependent Azimuthal Asymmetries in the Drell-Yan Process, Phys. Rev. Lett. 119, 112002; M. Kohl, for the MUSE Collaboration, The Muon Scattering Experiment (MUSE) at PSI and the proton radius puzzle, EPJ Web of Conferences 81, 02008 (2014). 